\title{
On the Borderline of Real and Complex Dynamics
}

\author{
Mikhail LyUBiCH
}

Mathematics Department and Institute for Math. Sciences

State Univ. of New York, Stony Brook, NY 11794, USA

\section{Introduction}

1.1. Overview. We will describc recent developments in several intimately related problems of complex and real one-dimensional dynamics: rigidity of polynomials and local connectivity of the Mandelbrot set, measure of Julia sets, and attractors of quasi-quadratic maps. A combinatorial basis for this study is provided by the Yoccoz puzzle. The main problem is to understand the geometry of the puzzle. Our main geometric result is that in the quadratic case its principal moduli grow linearly. Renormalization ideas are strongly involved in the discussion. The interplay between real and complex dynamics enlightens both. In the end we will briefly discuss a new geometric object which can be associated to a rational function, a hyperbolic orbifold 3-lamination.

1.2. Polynomial dynamics: Definitions and notation. For the reader's convenience and to fix the notations we will give here the definitions of some basic objects in holomporphic dynamics.

Let $P(z)=z^{d}+a_{1} z^{d-1}+\ldots+a_{d}$ be a monic polynomial of degree $d \geq 2$, and $P^{n}$ its $n$-fold iteratc. The basin of $\infty$ is the set of points escaping to $\infty$ : $D(\infty)=\left\{z: P^{n} z \rightarrow \infty\right\}$. The filled Julia set is its complement: $K(P)=\mathbf{C} \backslash D(\infty)$. The Julia set $J(P)$ is the common boundary of $D(\infty)$ and $K(P)$.

The Julia set (and the filled Julia set) is connected if and only if none of the critical points escapes to $\infty$. In this case there is a unique conformal map $R: D(\infty) \rightarrow\{z:|z|>1\}$, normalized by $R(z) \sim z$ as $z \rightarrow \infty$. Note that $R \circ P \circ R^{-1}: z \mapsto z^{d}$. The external rays and equipotentials of $P$ are defined as the $R$-preimages of the straight rays $\left\{r e^{i \theta}: 1<r<\infty\right\}$ and round circles $\left\{r e^{i \theta}: 0 \leq \theta \leq 2 \pi\right\}$.

A polynomial $P$ is called hyperbolic if the orbits of all critical points converge to attracting cycles in $\mathbf{C}$ or $\infty$. It is called postcritically finite if the orbits of all critical points are finite.

Two polynomials $P_{1}$ and $P_{2}$ are called topologically (conformally, quasi-conformally) conjugate if there is a homeomorphism (conformal isomorphism, quasiconformal map correspondingly) $h: \mathbf{C} \rightarrow \mathbf{C}$ such that $P_{1}=h^{-1} \circ P_{2} \circ h$.

\section{Rigidity Conjecture}

2.1. Our main object will be the quadratic family $P_{c}: z \mapsto z^{2}+c, c \in$ C. A key problem in the modern holomorphic dynamics is to classify quadratics up to topological conjugacy. Here is the main conjecture. 
QuAdratic Rigidity ConjeCture. Any nonhyperbolic quadratic polynomial $P_{b}$ is not conjugate to any other quadratic polynomial $P_{c}$.

Let us look closer at the meaning of this conjecture from the point of view of the bifurcation diagram in the parameter plane. The Mandelbrot set $M I$ is defined as the set of $c$ for which the Julia set $J\left(P_{c}\right)$ is connected. A component $H$ of int $M$ is called hyperbolic if it is filled with hyperbolic quadratics. The hyperbolic component contains one special point $c_{H}$, its center, where the critical point is periodic. The Rigidity Conjecture would assert that the Mandelbrot set splits into the following topological classes: hyperbolic components of int $M$ punctured at their centers, and single points.

A possible nonhyperbolic component $Q$ of int $M$ is called "quecr". It is still true that all polynomials within $Q$ are topologically conjugate (sce [L0], [MSS]). So the Rigidity Conjecture would imply absence of queer components, and hence density of hyperbolic maps in the quadratic family. This conjecture is a special casc of the so-called Fatou Conjecture (sec [F], p. 73, and discussion in [McM3]).

Quadratic Fatou Conjecture. Hyperbolic polynomials are dense in the quadratic family.

There is another famous conjecture due to Douady and Hubbard:

MLC ConjeCture. The Mandelbrot set is locally connected.

It turns out to be true, though not at all obvious, that this conjecture is stronger than both of the above (compare [DH1]). So we have the following implications:

MLC Conjecture $\Rightarrow$ Rigidity Conjecture $\Rightarrow$ Fatou Conjecture.

Though the Rigidity Conjecture is formally weaker than MLC, so far progress has been made simultanuously in both by means of the same ideas and methods (at least, outside the boundaries of the hyperbolic components). On the other hand, there is an ergodic approach to the Fatou Conjecture that may settle it before the other two (sce [MSS], [McM2]).

2.2. Copies of the Mandelbrot set and Douady-Hubbard renormalization. The Mandelbrot set contains many "little copies of itself" canonically homeomorphic to the whole set $M I$ but different from $M I$ (see [DH1], [D], [M1]). Each copy arises from a hypcrbolic component $H$ of $M$. A map $P_{c}$ (and the corresponding parameter value) is called Douady-Hubbard (DH) renormalizable if $c$ belongs to a little copy of the Mandelbrot set (we will sometimes say just "renormalizable" if it cannot be confused with a generalized notion from 3.6). If there are two nested Mandelbrot copies containing $c$, then $c$ is twice renormalizable, etc. In particular, we can classify the quadratics as finitely or infinitely $\mathrm{DH}$ renormalizable.

A copy $M^{\prime}$ is called maximal if it is not contained in any other copy. Let $\mathcal{M}$ denote the set of all maximal copies of $M$, and let $\sigma: \cup_{M I^{\prime} \in \mathcal{M}} M^{\prime} \rightarrow M$ be the map whose restriction onto any copy $M^{\prime} \in \mathcal{M}$ is the canonical homeomorpism onto $M$. To any infinitely renormalizable $c \in M$ wc can associate its $D H$ combinatorial type. This is a sequence $\tau(c)=\left[M_{1}, M_{2}, \ldots\right]$ of maximal copies $M_{n} \in \mathcal{M}$, defined by $\sigma^{n}(c) \in I_{n}$. Moreover, any such sequence is realized for some parameter valuc.

In 3.4 we will make clear the dynamical meaning of $\mathrm{DH}$ renormalization. 
2.3. Rigidity theorems. Let us start with a rigidity result that marked the beginning of a new stage in the field (and resolved a problem of monotonicity of topological entropy in the quadratic family, see [MT, Section 13], [DH3]). We will specify it for the quadratic family.

TheOREM 2.1 (Thurston). Any postcritically finite quadratic is rigid.

The next breakthrough was made in the work of Branner and Hubbard on cubic maps with one escaping critical point $[\mathrm{BH}]$, and Yoccoz's work on quadratics (see the discussion in [H], [L3] and [M2]). Again we will state only the quadratic result.

THEOREM 2.2 (YocCOZ). Any quadratic polynomial $P_{c}$ that is at most finitely renormalizable and has no attracting periodic points in the finite plane is rigid. Moreover, MLC holds at $c$.

(Note that quadratics with attracting periodic point are hyperbolic.) The following result of the author settles many infinitely renormalizable cases:

THEOREM 2.3 [L4]. There is a family $\mathcal{S} \subset \mathcal{M}$ of maximal Mandelbrot copies such that if $\tau(c)=\left[M_{1}, M_{2}, \ldots\right]$ with $M_{n} \in \mathcal{S}$ then $P_{c}$ is rigid. Moreover, MLC holds at $c$.

This family $\mathcal{S}$ is spccified by a property of sufficiently high combinatorial height (sec Section 3 for the definition). This condition becomes especially efficient on the real line, since it can be complemented by the following rigidity result of Sullivan (sce $[\mathrm{S}]$ and $[\mathrm{MvS}]$ ).

ThEOREM 2.4 (SUllivan). Let $c \in \mathbf{R}$ be an infinitely renormalizable parameter value, $\tau(c)=\left[M_{0}, M_{1}, \ldots\right]$ with all $M_{n}$ selected from a finite family of maximal copies of $M$. Then $P_{c}$ is rigid on the real line.

Combining the methods of Theorem 2.3 and Theorem 2.4 we obtain the following.

COROLLARY 2.5. Any nonhyperbolic real quadratic polynomial is rigid on the real line.

It follows that hyperbolic maps are dense on the real line. This result had been earlier announced by Swiatek [Sw], who approached it from the real point of view. A related rigidity result was also proven by McMullen (see [McM2]). The latter one asserts that any real non-hyperbolic quadratic polynomial is quasi-conformally rigid (that is, the quasi-conformal class of such a map consists of this map only).

Let us note that the main content of Theorem 2.3 is the so-called complex $a$ priori bounds, which yield much more than rigidity. We will discuss these issues later on.

\section{Combinatorial framework}

3.1. DH polynomial-like maps. Let $U^{\prime}$ and $U$ be two topological disks with $\operatorname{cl} U^{\prime} \subset$ $U$, and $f: U^{\prime} \rightarrow U$ be a holomorphic branched covering map. Such a map is called 
DH polynomial-like map. It is called $D H$ quadratic-like if $\operatorname{deg} f=2$. In this case we always put the critical point of $f$ at the origin 0 .

One can naturally define the filled Julia set of a polynomial-like map as the set of nonescaping points: $K(f)=\left\{z: f^{n} z \in U^{\prime}: n=0,1, \ldots\right\}$. The Julia set is defined as $J(f)=\partial K(f)$. These sets are connected if and only if all critical points are nonescaping, that is, belong to $K(f)$.

Actually one should view a polynomial-like map as a germ ncar its filled Julia set, so that the choice of the $U^{\prime}$ and $U$ is not canonical. Given a polynomiallike map $f: U^{\prime} \rightarrow U$, we can consider a fundamental annulus $A=U^{\prime} \backslash U$. Let $\bmod (f)=\sup \bmod A$, where $A$ runs over all fundamental annuli of $f$. The control of moduli of appropriate polynomial-like maps is a key issue of the renormalization theory (see $[\mathrm{S}]$, [McM2]).

If there is a quasi-conformal conjugacy $h$ between two polynomial-like maps $f$ and $g$ (near the filled Julia sets), with $\bar{\partial} h=0$ almost everywhere on the filled Julia set $K(f)$, then $f$ and $g$ arc called hybrid cquivalent. A hybrid class $\mathcal{H}(f)$ is the space of maps hybrid equivalent to $f$ modulo conformal equivalence. According to Sullivan, $\mathcal{H}(f)$ should be viewed as an infinitely dimensional Teichmüller space. In contrast with the classical Teichmüller theory this space has a preferred point: Any hybrid class of polynomial-like maps with connected Julia set contains a unique polynomial of the same degree (Straightening Theorem [DH2]). In particular, the hybrid classes of quadratic-like maps $z \mapsto z^{2}+c$ arc labcled by the points $c=$ $c(f) \in M$ of the Mandelbrot set.

Given a DH polynomial-like map with connected Julia set, we can define external rays and equipotentials near the filled Julia set by choosing some hybrid conjugacy to a polynomial (of course thesc curves are not uniquely defined).

3.2. Limbs. Let $P_{c}$ be a quadratic polynomial with both fixed points being repelling, and let $\alpha_{c}$ be the dividing fixed point, so that $J\left(P_{c}\right) \backslash\left\{\alpha_{c}\right\}$ is disconnected. There are finitely many external rays $R_{i}\left(\alpha_{c}\right)$ landing at $\alpha_{c}$, which arc cyclically permuted by $P_{c}$ with combinatorial rotation number $\rho\left(\alpha_{c}\right)=q_{c} / p_{c}$ (sce $\left.[\mathrm{H}]\right)$.

This rotation number can be easily read off from the position of $c$ at the Mandelbrot set. Let $b$ be the parabolic bifurcation point on the main cardioid of $M$ where $P_{b}^{\prime}\left(\alpha_{b}\right)=e^{2 \pi i q / p}$. The connected component of $M \backslash\{b\}$, which does not contain the origin, is called the primary $\operatorname{limb} L_{b}$ of $M$ with root at $b$. It turns out that if $c \in L_{b}$ then $\rho\left(\alpha_{c}\right)=q / p$. Similarly, given a hyperbolic component $H$ attached to the main cardioid, we can consider secondary limbs $L_{b}$ attached to $H$ at bifurcation points $b \in \partial H$.

We refer to a truncated limb if we remove from it a neighborhood of its root.

3.3. Yoccoz puzzle. Let $J\left(P_{c}\right)$ be connected with both fixed points being repclling. Let $E$ be an equipotential of $P_{c}$. The rays $R_{i}(\alpha)$ landing at $\alpha$ cut the domain bounded by $E$ into $p$ closed topological disks $Y_{i}^{(0)}, i=0, \ldots, p-1$, called puzzlc picces of zero depth.

Let us define puzzle pieces $Y_{i}^{(n)}$ of depth $n$ as the connected components of $f^{-n} Y_{k}^{(0)}$. They form a finite tiling of the neighborhood of $K(f)$ bounded by $f^{-n} E$. For every depth there is one puzzle piece containing the critical point. It is called critical and is labeled as $Y^{(n)} \equiv Y_{0}^{(n)}$. 
The Yoccoz puzzle provides us with the Markov family of puzzle pieces to play with. There are several different ways to do this: by means of the BrannerHubbard tableaux [BH], or by means of the Yoccoz $\tau$-function (unpublished), or by means of the principal nest and generalized renormalization ([LM], [L1]-[L4]), as will be described below.

3.4. Principal nest. Let us say that a map $f=P_{c}$ is immediately $D H$ renormalizable if the orbit of 0 does not escape $Y^{(1)}$ under iterates of $f^{p}$. In this case $c$ belongs to a copy of $M$ attached to the main cardioid.

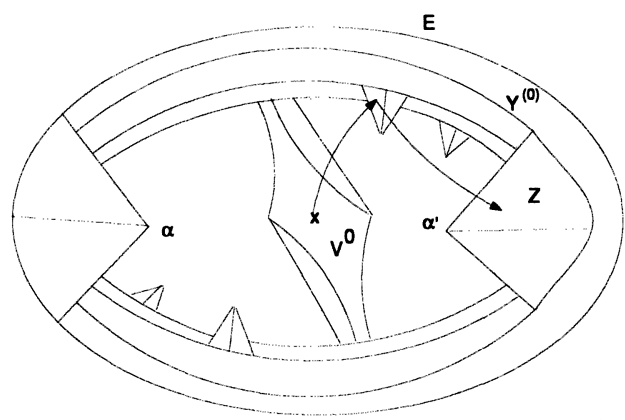

Figure 1. Puzzle.

If $P_{c}$ is not immediately renormalizable, then there is a $t$ such that $f^{t p} 0 \in Z$, where $Z$ is a noncritical puzzle piece of depth 1 attached to $\alpha^{\prime}=-\alpha$ (see Figure 1). Then let us construct the (short) principal nest $V^{1} \supset V^{2} \supset \ldots$ of puzzle pieces in the following way.

Let $t$ be the first moment when $f^{t p} 0 \in Z$. Then let $V^{0} \ni 0$ be the pull-back of $Z$ along the orbit $\left\{f^{n} 0\right\}_{0}^{t p}$, that is, the critical puzzle piece such that $f^{t p} V^{0}=Z$. Further, let us define $V^{n}$ as the pull back of $V^{n-1}$ corresponding to the first return of the critical point 0 back to int $V^{n}$. Then we have a double branched covering $g_{n}=f^{l(n)}: V^{n} \rightarrow V^{n-1}$, where $l(n)$ is the corresponding return time.

Let us call a return to level $n-1$ central if $g_{n} 0 \in V^{n}$. If we have several consequtive central returns, we refer to a central cascade of puzzle pieces. Let $\chi(f)$ denote the number of noncentral levels in the principle nest. We call it the height of $f$. In other words, $\chi(f)$ is the number of different quadratic-like maps in the sequence $g_{n}$ (recall that we think of quadratic-like maps as germs, see Section 3.1). The height is finite if and only if $f$ is renormalizable.

Renormalizable maps can be easily recognized in terms of the principal nest. Namely, a map $f$ is $D H$ renormalizable if and only if it is either immediately renormalizable, or there is a level $N$ such that the critical point 0 does not escape $V^{N}$ under iterates of $g_{N}$.

In the immediately renormalizable case a little enlargement $U^{\prime} \supset Y^{(1)}$ provides us with a quadratic-like map $f^{p}: U^{\prime} \rightarrow U$ with non-escaping critical point (compare [DH1], [M2]). When $f$ is renormalizable, but not immediately, then $g_{N}: V^{N} \rightarrow V^{N-1}$ is a quadratic-like map with non-escaping critical point. In both cases the corresponding quadratic-like map up to conformal equivalence is called the $D H$ renormalization $R f$ of $f$. Note that the hybrid class of $R P_{c}$ is labeled by $\sigma(c) \in M$, where $\sigma$ is the map from Section 2.2. 
Now we can study $R f$ by the same means as $f$ : cut the Julia set by external rays, consider the Yoccoz puzzle and the principle nest, etc. If $R f$ is also renormalizable, we will repeat the procedurc, and so on.

In such a way we obtain a sequence of renormalizations $R^{m} f$ and the corresponding principal nests of puzzle picces:

$$
\ldots \supset Y^{(m, 1)} \supset V^{m, 0} \supset V^{m, 1} \supset \ldots \supset V^{m, t(m)} \supset V^{m, t(m)+1} \supset \ldots
$$

We truncate this nest at a DH renormalizable level. In the immediately renormalizable case this nest is reduced to one piece $Y^{(m, 1)}$. In the finitely renormalizable case the nest corresponding to the last renormalization is infinite.

Let $A^{m, n}=V^{m, n-1} \backslash V^{m, n}$ denote the principal nest of annuli.

3.5. Generalized polynomial-like maps. Let $\left\{U_{i}\right\}$ be a finite or countable family of topological discs with disjoint interiors compactly contained in a topological disk $U$. We call a map $g: \cup U_{i} \rightarrow U$ a (generalized) polynomial-like map if $g: U_{i} \rightarrow U$ is a branched covering of finite degree which is univalent on all but finitely many $U_{i}$. The DH polynomial-like maps correspond to the case of a single disk $U_{0}$. All concepts introduced beforc for $\mathrm{DH}$ polynomial-like maps can be readily cxtended to the generalized situation: The filled Julia set $K(g)$ is the set of all non-cscaping points, the Julia set $J(g)$ is its boundary, etc. Let us say that a polynomial-like map $g$ is of finite type if its domain consists of finitely many disks $U_{i}$.

Generalized Straightening Theorem. Any polynomial-like map of finite type is hybrid equivalent to a polynomial with the same number of nonescaping critical points.

Let us call a (gencralized) polynomial-like map a (generalized) quadratic-likc map if it has a single (and nondegenerate) critical point. In such a casc we will always assume that 0 is the critical point, and label the discs $U_{i}$ in such a way that $U_{0} \ni 0$.

3.6. Generalized renormalization. Philosophically the dynamical renormalization is the first return map to an appropriate picce of the space considered up to conjugacy. Let us make this precise in our quadratic-like sctting.

Let $f: \cup U_{i} \rightarrow U$ bc a generalized quadratic-like map, and $V \ni 0$ bc a topological disk satisfying the following property: $f^{n}(\partial V) \cap V=\emptyset, n=1,2, \ldots$ Then the first return map $g: \cup V_{i} \rightarrow V$ has the following structure: it is defined on a union of disjoint topological disks $V_{i}$ compactly contained in $V$, and univalently maps all of them except the critical one onto $V$. The critical disk $V_{0}$ (if it exists) is two-to-one mapped onto $V$. Morcover, if the orbit of 0 under itcrates of $f$ infinitely many times visits $V$ then it is nonescaping under iterates of $g$.

Let us restrict $g$ on the union of domains $V_{i}$ visited by the critical orbit. We call this map considered up to affine rescaling the gencralized renormalization $T_{V} f$ of $f$ on the domain $V$.

Let us define the $n$-fold generalized renormalization of a DH quadratic-likc map $f, T^{n} f \equiv g_{n}: \cup V_{i}^{n} \rightarrow V^{n-1}$, as the generalized renormalization of $f$ on the piece $V^{n-1}$ of the principal nest. This sequence of renormalizations is our key to understanding geometry of the map. 
3.7. Special families of Mandelbrot copies. Note that the height function $\chi\left(P_{c}\right)$ is constant over any copy $M^{\prime}$ of the Mandelbrot set. So we can use the notation $\chi\left(M^{\prime}\right)$. Let $\mathcal{S} \subset \mathcal{M}$ be a family of maximal copies of the Mandelbrot set. Let us call it special if it satisfies the following property: for any truncated secondary limb $L$ there is a height $\chi_{L}$ such that $\mathcal{S}$ contains all copies $M^{\prime} \subset L$ of the Mandelbrot set with $\chi\left(M^{\prime}\right) \geq \chi_{L}$.

Let $f$ be an infinitely DH renormalizable quadratic-like map with $\tau(f)=$ $\left[M_{1}, M_{2}, \ldots\right]$. Let us say that it is of $\mathcal{S}$-type if $M_{n} \in \mathcal{S}, n=0,1, \ldots$

\section{Geometry of the puzzle}

The main geometric problem is to gain control of sizes and shapes of puzzle pieces. To this end we need to bound the moduli of the annuli $A^{n}=V^{n-1} \backslash V^{n}$ in the principal nest (we skip the first index $m$ when we work within a fixed renormalization level). The following lemma allows us to begin.

LEMMA 4.1 (INITIAL MODULUS). Let $P_{c}$ be a quadratic polynomial with $c$ ranging over a truncated secondary limb $L^{\text {tr }}$. Then $\bmod \left(A^{1}\right) \geq \nu>0$ with $\nu$ depending only on $L^{t r}$.

The rough reason is that configuration of external rays of $P_{c}$ has bounded geometry when $c$ ranges over a truncated limb. The next theorem is our main geometric result [L4]:

TheOREM 4.2 (MODULI GROWTH). Let $n(k)$ count the noncentral levels in the short principle nest. Then $\bmod A_{n(k)+1} \geq C k$, where $C$ depends only on $\bmod A_{1}$.

The proof is based upon combinatorial and geometric analysis of the cascade of generalized renormalizations. The above two results yield the complex a priori bounds for maps of special type:

THEOREM 4.3 (A PRIORI BOLNDS). There is a special family $\mathcal{S}$ of Mandelbrot copies with the following property. If $P$ is an infinitely renormalizable quadratic polynomial of $\mathcal{S}$-type, then $\bmod \left(R^{n} P\right) \geq \mu(\mathcal{S})>0$.

Proof. Let us fix a big $Q>0$. Let $f$ bc a quadratic-like map with $\tau(f)=$ $\left[M_{0}, M_{1}, \ldots\right]$, where the Mandelbrot copy $M_{n}$ belongs to a truncated limb $L_{n}$. Assume $\bmod f>Q$. By Lemma 4.1 the modulus of the first annulus in the long principal nest is definite: $\bmod A^{0,1} \geq C(Q) \nu\left(L_{0}\right)>0$. If the height of $M_{n}$ is sufficiently big (depending on $L_{n}$ ), then by Theorem 4.2 the modulus of the last annulus $A^{0, t(0)+1}$ of the short nest will be at least $Q$. Hence $\bmod (R f) \geq Q$, and we can repeat the argument.

\section{Rigidity and pullback argument}

Let us call two infinitely renormalizable polynomials combinatorially equivalent if they have the same type. The topological classes are clearly contained in the combinatorial ones. So the Rigidity Conjecture would follow if we knew that the combinatorial classes are single points (which is actually equivalent to MLC for 
infinitely renormalizable quadratics). A well-known approach to this problem is based upon the following remark: if all polynomials within the combinatorial class $\mathcal{C}_{b}$ of $P_{b}$ are quasi-conformally conjugate, then this class is reduced to a single point $\{b\}$. Indeed, combinatorial classes are clearly closed. On the other hand, quasiconformal classes are open unless they are single points (by varying of Beltrami differentials). Hence $\mathcal{C}_{b}$ must be a single point.

Let us have a finite family $\mathcal{L}$ of truncated secondary limbs $L_{i}$. Denote by $\mathcal{S}(\mathcal{L}, \chi) \subset \mathcal{M}$ all Mandelbrot copics contained in $\cup L_{i}$ whose height is at least $\chi$. The following result implics the Rigidity Theorem 2.3.

THEOREM 5.1. There is a $\chi$ depending on $\mathcal{L}$ such that any two quadratic-like maps $f$ and $\tilde{f}$ of the same $\mathcal{S}(\mathcal{L}, \chi)$-type are quasi-conformally conjugate.

The method we usc for the proof is called "the pullback argument". The idea is to start with a qc map respecting some dynamical data, and then pull it back so that it will respect some new data on each step. In the end it becomes (with some luck) a qc conjugacy. This method originated in the works of Thurston (sce [DH3]), McMullen (see [McM1], Prop. 8.1) and Sullivan (see [MvS]) (perhaps, it can actually be tracked further down, in the setting of Kleinian groups). Then it was developed in several other works, for more complicated combinatorics (sec Kahn $[\mathrm{K}]$ and Swiatek $[\mathrm{Sw}]$ ). In particular, using this method, Jercmy Kahn gave a new proof of the Yoccoz Rigidity Theorem 2.2.

Our way is to pull back through the cascade of generalized renormalizations. The geometric bounds of the previous section are the crucial ingredients of the argument. The linear growth of moduli (Theorem 4.2) kecps the dilatation of pullbacks bounded until the next DH renormalization level, while complex a priori bounds (Theorem 4.3) allow us to penetrate through the next level.

Another method to prove Theorem 2.3 is to transfer the geometric results of Section 4 into the parameter plane (in preparation).

\section{Real dynamics}

6.1. Scaling factors. A $C^{3}$-map $f:[-1,1] \rightarrow[-1,1]$ is called quasi-quadratic if it has a negative Schwarzian derivative and a single nondegenerate critical point. Assume that this map has a fixed point $\alpha$ with negative multiplier (otherwise it is dynamically trivial), and let $\alpha^{\prime}$ be the dynamically symmetric point: $f \alpha=f \alpha^{\prime}$. Let $I_{0}=\left[\alpha, \alpha^{\prime}\right]$. Assume also that the critical point is recurrent. Then we can consider the first return of the critical point to $I^{0}$, and pull $I^{0}$ along the corresponding orbit. This gives us an interval $I^{1} \subset I^{0}$. Now we can consider the first return to $I^{1}$ and the corresponding pullback. and so on. In such a way we construct the real counterpart of the principal nest: $I^{0} \supset I^{1} \supset \ldots$. Moreover, all combinatorial notions such as central returns, DH renormalization, generalized renormalization, etc. are readily transferred to the real case (in the case of a real quadratic polynomial $P_{c}, c \in \mathbf{R}$ they arc just the "real traces" of the corresponding complex notions).

Let $|J|$ denote the length of an interval $J$. Let us define the scaling factors $\lambda_{n}$ as the ratios $\left|I^{n}\right| /\left|I^{n-1}\right|$. The real counterpart of Theorem 4.2 is the following result: 
THEOREM 6.1 (GEOMETRY DECAYS). Let $n(k)$ count noncentral returns in the principal nest. Then $\lambda_{n(k)+1} \leq C q^{k}$, where $C>0$ and $q<1$ depend only on the initial geometry of $f$.

In the quadratic-like case this result follows from Theorem 4.2. In the DH nonrenormalizable case it was proven in [L2] by passing to limits of generalized renormalizations and proving that they are generalized quadratic-like maps. In general we prove Theorem 6.1 by extending $f$ to the complex plane so that it is asymptotically conformal near the real line, and using the "quadratic-like" technique with exponentially small errors [L5].

The first application of this geometric result was to the problem of attractors (see the next section). Other applications are to come.

6.2. Fibonacci maps. These are important examples satisfying some extremal combinatorial properties. Though they are nonrenormalizable in the usual sense, they can be treated as infinitely renormalizable in the generalized sense described above. The domains of these renormalizations $T^{n} f=g_{n}: V_{0}^{n} \cup V_{1}^{n} \rightarrow V_{0}^{n-1}$ consist of two puzzle pieces on all levels.

The geometric properties of quasi-quadratic Fibonacci maps from the renormalization point of view were studied in [LM], where the following asymptotic formula for the scaling factors was proven:

$$
\lambda_{n} \sim a\left(\frac{1}{2}\right)^{n / 3},
$$

and hence the geometry of the postcritical Cantor set $\omega(0)$ is exponentially decaying.

Let $\mathcal{F}_{d}$ denote the class of $S$-unimodal Fibonacci maps with critical point 0 of type $x^{d}$. In contrast with (6.1), the postcritical set of an $f \in \mathcal{F}_{d}$ has bounded geometry for $d>2$ : the scaling factors stay away from 1 and 0 . In this case the renormalization approach and complex bounds of [LM], [L2] combined with Sullivan's [S] or McMullen's [McM4] arguments lead to the following result:

THEOREM 6.3. Let $d$ be an even integer. Then there is only one real Fibonacci polynomial $x \mapsto x^{d}+c$ of degree $d$ (rigidity). If $d>2$ then the generalized renormalizations $T^{n} f$ of any Fibonacci map $f \in \mathcal{F}_{d}$ converge to a cycle of period two independent of the initial map $f$.

Keller and Nowicki $[\mathrm{KN}]$ have studied the real geometry of the higher degree Fibonacci maps, and obtained the following bounds:

$$
C_{1} / d \leq \lambda_{n}(f) \leq C_{2} / d,
$$

where the constants are universal in degree. So $\lambda_{n}^{d}$ is bounded from both sides uniformly on $d$. This indicates a possibility of a new universality phenomenon near the critical value:

Problem. Study the asymptotical shape of the puzzle pieces $f V^{n}$ as $d \rightarrow \infty$. 
Note that in the quadratic case the Fibonacci renormalization theory implies that the shape of high level puzzle pieces near the critical point imitates the filled Julia set of $z \mapsto z^{2}-1$ [L5] (sce Figure 2).

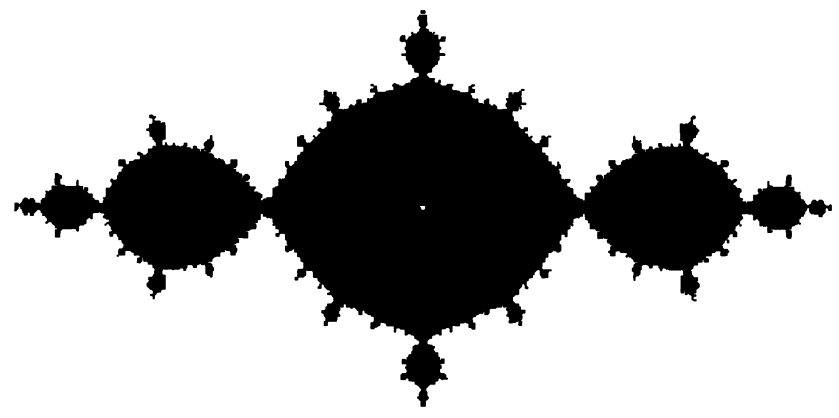

Figure 2. A puzzle piece for the degree 2 Fibonacci map.

\section{Attractors and measure of the Julia set}

7.1. Quasi-quadratic case. Our first application of geometric Theorcm 6.1 was the following result, which resolved Milnor's problem on attractors [N3] for quasiquadratic maps:

Theorem 7.1 [L2]. Let $f:[-1,1] \rightarrow[-1,1]$ be a $D H$ nonrenormalizable quasiquadratic map without attracting fixed points. Then $\omega(x)=\left[f 0, f^{2} 0\right]$ for Lebesgue almost all $x \in[-1,1]$.

A theoretical alternative would be a "wild" Cantor attractor $A=\omega(0)$ attracting almost all $x \in[-1,1]$ (sce [L3] for a survey on this problem, and Section 7.2 below).

The complex counterpart of Theorem 7.1 is the following theorem by the author [L1] and M. Shishikura (unpublished). The cubic case with one escaping critical point had been earlier treated by McMullen (see $[\mathrm{BH}]$ ).

THEOREM 7.2. If $P_{c}$ is at most a finitely renormalizable quadratic polynomial without irrational neutral periodic points then mes $J\left(P_{c}\right)=0$.

7.2. Higher degree Fibonacci maps. When the work [LM] on quasi-quadratic Fibonacci maps was done, the author suggested an approach to the problem of attractors and the measure problem for higher degree Fibonacci maps. It was based upon consideration of a random walk on the principal nest of annuli with transition maps corresponding to the generalized renormalization. Drift to the left for this random walk corresponds to existence of a "wild" measure-theoretic attractor in the real setting, and positive measure of the Julia sct in the complex setting.

Together with F. Tangerman, the author carried out a computer experiment based on this random walk approach to figurc out if there are "wild attractors" in higher degrees. The experiment gave the positive answer already for degree 6 . Recently this method has been carricd out rigorously:

THEOREM 7.3 [BKNS]. If $d$ is sufficiently big, then any Fibonacci map $f \in \mathcal{F}_{d}$ has a Cantor attractor: $\omega(x)=\omega(0)$ for Lebesgue almost all $x$ (even though this map is topologically transitive on $\left.\left[f 0, f^{2} 0\right]\right)$. 
A similar experiment in the complex plane carried out jointly with S. Sutherland has recently shown positive measure of the Julia set for the degree 32 Fibonacci map. Nowicki and van Strien have proven this as a rigorous result:

THEOREM 7.4 [SN]. The Fibonacci polynomial $z \mapsto z^{d}+c$ of sufficiently high degree has a Julia set of positive measure.

The expcriment and the proof are based upon the same random walk idea and the Fibonacci renormalization theory (Theorem 6.3). The amazing new geometric ingredient of $[\mathrm{SN}]$ is a rigorous analysis of the sizes and shapes of the puzzle pieces of the principal nest (compare Figure 3).

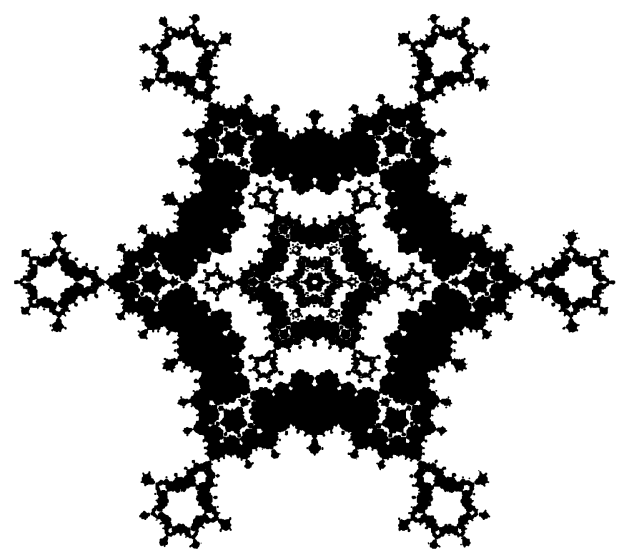

Figure 3. Pricipal nest for the degree 6 Fibonacci map.

Problem. Prove that there is a critical exponent $\delta$ such that Fibonacci maps of power higher than $\delta$ have a Cantor attractor, whereas those of smaller degree do not. Prove the same result for the measure of the Julia set (with a different exponent).

There is a remarkable connection between the problem of measure of the Julia set and the rigidity problem. Namely: If the Julia set $J(f)$ has zero measure then $f$ is quasi-conformally rigid [MSS]. Theorems 6.3 and 7.4 show, however, that thesc problems are not cquivalent.

\section{Rigidity and hyperbolic orbifold 3-laminations}

A great insight in the theory of Kleinian groups comes from the third dimension (see $[\mathrm{Th}]$ ), which allows one to relate hyperbolic geometry to the action at infinity. This gives powerful tools for the rigidity problems and the Ahlfors measure problem. So far nothing like this has appeared in holomorphic dynamics (though 3D analogy plays an important role in the work of McMullen, see [McM4]). Yair Minsky and the author have recently made an attempt to fill in this gap [LMin]. It was inspired by Sullivan's work on Riemann surface laminations which play the rolc of Ricmann surfaces associated to Klcinian groups (see [MvS], [S]).

Let me briefly outline our construction. Let $f$ bc a rational function. Consider the natural extension $\hat{f}: \mathcal{N}_{f} \rightarrow \mathcal{N}_{f}$, where $\mathcal{N}_{f}$ is the space of backward orbits of 
$f$. This space contains a regular part $\mathcal{R}_{f}$ that is decomposed into a union of leaves with a natural conformal structure. All these leaves are either hypcrbolic or parabolic planes.

Type Problem. Are there hyperbolic leaves except Siegel disks and Herman rings?

Parabolic leaves are conformally equivalent to $\mathbf{C}$, and hence bear an intrinsic affine structure preserved by dynamics. Unfortunately, this structure is not necessarily continuous in the transversal direction. To make it continuous one should strengthen the topology of $\mathcal{R}_{f}$. To get a reasonable object one then should complete $\mathcal{R}_{f}$. This procedure adds some singular leaves, with orbifold affine structure. An object that we build in such a way can be called an affinc orbifold lamination.

The next step is to attach hyperbolic orbifold 3-leaves to these affine 2leaves. This gives us a hyperbolic orbifold 3-lamination $\mathcal{H}_{f}$. The map $\hat{f}$ can be extended to this space as hypcrbolic isometries on the leaves, and it acts properly discontinuously on $\mathcal{H}_{f}$. The final step is to takc the quotient $\mathcal{H}_{f} / \hat{f}$. This hypcrbolic orbifold 3-lamination is our candidate for a role similar to that which hyperbolic 3 -manifolds play in the theory of Kleinian groups.

Having such an object in hand, we can define its convex corc. A map $f$ is called convex cocompact if this convex hull is compact.

THEOREM 8.1. Let $f$ be a postcritically finite rational function. Then the hyperbolic orbifold 3-lamination $\mathcal{H}_{f} / f$ is convex cocompact.

This leads to a three-dimensional proof of the Thurston Rigidity Theorem 2.1, which follows the same lines as the proof of the Mostow rigidity theorem, with the substitution of "lamination" for "manifold".

Acknowledgement. I thank John Milnor and Curt McMullen for many uscful comments on the manuscript. I also thank Scott Sutherland and Brian Yarrington for making the computer pictures.

\section{References}

[BH] B. Branner and J. H. Hubbard, The iteration of cubic polynomials, Part II, Acta Math. 169 (1992), 229-325.

[BKNS] H. Bruin, G. Keller, T. Nowicki, and S. van Strien, Absorbing Cantor sets in dynamical systems: Fibonacci maps, preprint IMS Stony Brook, 1994/1.

[D] A. Douady, Chirurgie sur les applications holomorphes, Proc. Internat. Congress Math. Berkeley, 1 (1986), 724-738.

[DH1] A. Douady and J. H. Hubbard, Étude dynamique des polynômes complexes, Publ. Math. Orsay, 84-02 and 85-04.

[DH2] A. Douady and J. H. Hubbard, On the dynamics of polynomial-like maps, Ann. Sci. École Norm. Sup. (4), 18 (1985), 287-343.

[DH3] A. Douady and J. H. Hubbard, A proof of Thurston's topological characterization of rational functions, Acta Math. 171 (1993), 263-297.

[F] P. Fatou, Sur les équations fonctionnelles, Bull. Soc. Math. France, 48 (1990), 33-94.

[H] J. H. Hubbard, Local connectivity of Julia sets and bifurcation loci: Three theorems of J.-C. Yoccoz, Topological Methods in Modern Math., A Symposium in Honor of John Milnor's 60th Birthday, Publish or Perish, Houston, TX, 1993. 
[K] J. Kahn, Holomorphic removability of Julia sets, manuscript in preparation.

[KN] G. Keller and T. Nowicki, Fibonacci maps re(al)visited, Ergod. Th. \& Dynam. Syst., 15 (1995), 99-120.

[L0] M. Lyubich, Some typical properties of the dynamics of rational maps, Russian Math. Surveys 38 (1983), 154-155.

[L1] M. Lyubich, On the Lebesgue measure of the Julia set of a quadratic polynomial, preprint IMS at Stony Brook, 1991/10.

[L2] M. Lyubich, Combinatorics, geometry and attractors of quasi-quadratic maps, Ann. Math. 140 (1994), 347-404.

[L3] M. Lyubich, Milnor's attractors, persistent recurrence and renormalization, Topological Methods in Modern Mathematics, A Symposium in Honor of John Milnor's 60th Birthday, Publish or Perish, Houston, TX, 1993.

[L4] M. Lyubich, Geometry of quadratic polynomials: Moduli, rigidity and local connectivity, preprint IMS Stony Brook, 1993/9.

[L5] M. Lyubich, Teichmüller space of Fibonacci maps, preprint IMS Stony Brook, 1993/12.

[LM] M. Lyubich and J.Milnor, The unimodal Fibonacci map, preprint IMS Stony Brook, 1991/15; J. Amer. Math. Soc., 6 (1993), 425-457.

[LMin] M. Lyubich and Y.Minsky, Laminations in holomorphic dynamics, preprint IMS at Stony Brook, 1994/20.

[MSS] R. Mañé, P. Sad, and D. Sullivan, On the dynamics of rational maps, Ann. Sci. École Norm. Sup. (4), 16 (1983), 193-217.

[McM1] C. McMullen, Families of rational maps, Ann. Math., 125 (1987), 467-493.

[McM2] C. McMullen, Complex dynamics and renormalization, Annals of Math. Studies, 135, Princeton Univ. Press, 1994.

[McM3] C. McMullen, Frontiers of holomorphic dynamics, Bulletin AMS, 331 (1994), 155-172.

[McM4] C. McMullen, Renormalization and 3-manifolds which fiber over the circle, preprint, 1994.

[MvS] W. de Melo and S. van Strien, One dimensional dynamics, Springer-Verlag, Berlin and New York, 1993.

[M1] J. Milnor, Self-similarity and hairiness in the Mandelbrot set, Computers in geometry and topology, Lecture Notes in Pure and Appl. Math., 114 (1989), 211257.

[M2] J. Milnor, Local connectivity of Julia sets: Expository lectures, preprint IMS Stony Brook, 1992/11.

[M3] J. Milnor, On the concept of attractor, Comm. Math. Phys, 99 (1985), 177-195, and 102 (1985), 517-519.

[MT] J. Milnor and W.Thurston, On iterated maps of the interval, Dynamical Systems, Proc. U. Md., 1986-87, ed. J. Alexander, Lecture Notes in Math., 1342 (1988), 465-563.

[SN] S. van Strien and T. Nowicki, Polynomial maps with a Julia set of positive Lebesgue measure: Fibonacci maps, preprint IMS at Stony Brook 1994/3.

[Sw] G. Swiatek, Hyperbolicity is dense in the real quadratic family, preprint IMS at Stony Brook 1992/10.

[S] D. Sullivan, Bounds, quadratic differentials, and renormalization conjectures, Amer. Math. Soc. Centennial Publications. 2: Mathematics into Twenty-first Century (1992).

[Th] W. Thurston, The geometry and topology of three-manifolds, preprint, 1979. 